\title{
Autobiographical narratives relate to Alzheimer's disease biomarkers in older adults
}

Rachel F. Buckley ${ }^{\mathrm{a}, \mathrm{b}}$ *, Michael M. Saling ${ }^{\mathrm{a}, \mathrm{b}}$, Muireann Irish ${ }^{\mathrm{c}, \mathrm{d}}$, David Ames ${ }^{\mathrm{e}, \mathrm{f}}$, Christopher

C. Rowe ${ }^{\mathrm{g}, \mathrm{h}}$, Victor L Villemagne ${ }^{\mathrm{g}, \mathrm{h}}$, Nicola T. Lautenschlager ${ }^{\mathrm{f}, \mathrm{i}}$, Paul Maruff ${ }^{\mathrm{b}, \mathrm{j}}$, S. Lance Macaulay $^{\mathrm{k}}$, Ralph N. Martins, m, n, Cassandra Szoeke ${ }^{\mathrm{e}}$, Colin L. Masters ${ }^{\mathrm{b}}$, Stephanie R. Rainey-Smith, m, Alan Rembach ${ }^{\mathrm{b}}$, Greg Savage ${ }^{\mathrm{o}}$, Kathryn A. Ellis ${ }^{\mathrm{b}, \mathrm{f}}$ and the Australian Imaging Biomarkers and Lifestyle Study of Ageing (AIBL) Research Group

${ }^{a}$ Melbourne School of Psychological Sciences, University of Melbourne, Melbourne, Australia

${ }^{b}$ The Florey Institute of Neuroscience and Mental Health, University of Melbourne, Melbourne, Australia

${ }^{c}$ Neuroscience Research Australia, Sydney, Australia

${ }^{d}$ School of Psychology, the University of New South Wales, Kensington, Sydney, NSW

${ }^{e}$ National Aging Research Institute, Melbourne, Australia

${ }^{f}$ The Academic Unit for Psychiatry of Old Age, St. Vincent's Health, Department of Psychiatry, University of Melbourne

${ }^{g}$ Department of Nuclear Medicine and Centre for PET, Austin Health, Heidelberg, Australia

${ }^{h}$ The Department of Medicine, Austin Health, University of Melbourne, Melbourne, Australia

${ }^{i}$ School of Psychiatry and Clinical Neurosciences and West Australian Centre for Health \& Ageing, University of Western Australia

${ }^{j}$ Cogstate Ltd, Melbourne, Australia

${ }^{k}$ Commonwealth Scientific Industrial Research Organization Preventative Health Flagship, Parkville, Australia

${ }^{l}$ Centre of Excellence for Alzheimer's Disease Research and Care, School of Medical Sciences, Edith Cowan University, Western Australia, Australia

${ }^{m}$ Sir James McCusker Alzheimer's Disease Research Unit (Hollywood Private Hospital), Perth, Western Australia, Australia

${ }^{n}$ School of Psychiatry and Clinical Neurosciences, University of Western Australia, Perth, Western Australia, Australia

${ }^{\circ}$ ARC Centre of Excellence in Cognition and its Disorders, Macquarie University, Sydney, Australia

Running title: AD biomarkers relate to ABM narratives

*Corresponding author: Rachel F. Buckley, B(Sc) Hons, Melbourne School of Psychological Sciences, Redmond Barry Building, University of Melbourne, Victoria, Australia, 3010, Tel: +613 8344 4297, Fax: +613 9347 6618, Email: rachel.buckley@unimelb.edu.au. 


\section{Abstract}

Background: Autobiographical memory (ABM), personal semantic memory (PSM), and autonoetic consciousness are affected in individuals with mild cognitive impairment (MCI) but their relationship with Alzheimer's disease (AD) biomarkers are unclear.

Methods: Forty-five participants (healthy controls $(\mathrm{HC})=31, \mathrm{MCI}=14$ ) completed the Episodic ABM Interview and a battery of memory tests. Thirty-one (HC=22, MCI=9) underwent $\beta$-amyloid positron emission tomography (PET) and magnetic resonance (MR) imaging. Fourteen participants ( $\mathrm{HC}=9, \mathrm{MCI}=5)$ underwent one imaging modality.

Results: Unlike PSM, ABM differentiated between diagnostic categories but did not relate to AD biomarkers. PSM was related to neocortical $\beta$-amyloid burden after adjusting for age and APOE $\varepsilon 4$. Autonoetic consciousness was not associated with $\mathrm{AD}$ biomarkers, and was not impaired in MCI.

Conclusion: ABM was impaired in MCI participants but was not related to neocortical amyloid burden, suggesting that personal memory systems are impacted by differing disease mechanisms, rather than being uniformly underpinned by $\beta$-amyloid. Episodic and semantic $\mathrm{ABM}$ impairment represent an important $\mathrm{AD}$ prodrome.

Key words: Autobiographical memory, $\beta$-amyloid, hippocampal volume, mild cognitive impairment 


\section{Introduction}

Subjective experiences of memory dysfunction are of growing importance in research on pathological aging (Sperling et al., 2010), particularly because they hold promise for very early preclinical detection of dementia of the Alzheimer's type (DAT). At present, mild cognitive impairment (MCI) is commonly classified on the basis of neuropsychological assessment with an emphasis on episodic memory dysfunction (Petersen et al., 1999). Clinical criteria for MCI also require the presence of a subjective memory complaint, or, in some cases, an observation of change from an informant. This is usually described in general terms and is elicited within the context of a routine clinical history (Petersen et al., 1999; Winblad et al., 2004). Importantly, there is no definitive framework to guide clinicians' exploration of subjective memory symptomatology. There is, however, an emerging interest in developing and refining the clinical evaluation of memory symptoms on presentation (Jessen et al., 2014).

There is no global consensus on the measurement of subjective memory complaints in MCI. Studies investigating subjective memory complaints predominantly rely on questionnaires that probe responses to itemized lists of circumstances where memory lapses can occur, such as the Memory Assessment Clinics Questionnaire, or MAC-Q (Buckley et al., 2013). Direct evaluation of memory personally relevant episodic or semantic knowledge, arguably the archetypical expression of subjective memory, is not typically included in the mainstream diagnostic approach to MCI, perhaps due to the difficulty in verifying the response. Personal memory encompasses autobiographical memory (ABM), personal semantic memory (PSM), and autonoetic consciousness. ABM refers to the recollection of highly contextualised individual experiences (Wheeler et al., 1997), involving remembering the details of the event as they took place within a spatiotemporal and emotional context (Irish et al., 2011a; 2011b). Autonoetic consciousness represents the rich accompanying re- 
experiencing of the event, and the mental capacity to travel forwards and backwards in time (Wheeler et al., 1997). Personal semantic memory (PSM), on the other hand, refers to personally relevant knowledge or facts about the individual (Robinson \& Swanson, 1990). For instance, an individual's recall of a birthday party will be characterised by the rich spatiotemporal and emotional ABM detail, and perhaps intensified by the autonoetic reexperiencing or reliving of the event in the mind's eye. In addition, personal semantic knowledge will be accessible to the individual, for example, of their birth date or the names of the individuals who attended. The ABM-PSM system as a cognitive entity is underpinned by a neural network involving medial and lateral temporal, prefrontal, and posterior parietal cortices (for a review see Svoboda et al. 2006). There is also growing evidence for a loss of ABM-PSM function in individuals with MCI and DAT (Fraser et al., 2008; Hou et al., 2005; Irish et al., 2011a; Irish et al., 2010; Leyhe et al., 2009). In our view, this provides a promising foundation on which to investigate the nature of subjective memory at a preclinical stage.

In individuals with DAT, poor ABM performance is associated with bilateral medial temporal atrophy and anterolateral temporal neocortex. PSM, on the other hand, is associated with atrophy in the temporal neocortex, often bilaterally (Gilboa et al., 2005). Measures of new learning and retention are associated with decrements in entorhinal and hippocampal volume in healthy older individuals and in MCI (Mormino et al., 2009). Despite extensive work on new learning (Kantarci et al., 2012; Lim et al., 2013a; 2013b; Mormino et al., 2009; Pike et al., 2011; Rentz et al., 2011) no studies have investigated the relationship between ABM, PSM and autonoetic consciousness, and AD biomarkers such as neocortical $\beta$-amyloid burden, brain atrophy and apolipoprotein E $\varepsilon 4$ ( $A P O E \varepsilon 4$ ), to the best of our knowledge. While this study will primarily on ABM and PSM, measures of new learning will also be included because of their they play a major current role in the current diagnosis of MCI and 
their relationship with neurobiomarkers in the literature will be summarised briefly. Neuroimaging studies of healthy elderly adults also suggest that a relationship exists between poorer performance on new learning tasks and increased brain $\beta$-amyloid load both crosssectionally (Kantarci et al., 2012; Rentz et al., 2011) and longitudinally (Lim et al., 2013b). This relationship is complex; some studies report a low to moderate effect size (Kantarci et al., 2012; Mormino et al., 2009), and others report no relationship (Pike et al., 2011). Carrying the APOE $\varepsilon 4$ allele might also play a mediating role in this relationship (Kantarci et al., 2012; Lim et al., 2013a; Pike et al., 2011).

The first aim of this study was to determine the extent to which neocortical $\beta$ amyloid, brain atrophy and APOE \&4, influences ABM, PSM, and autonoetic consciousness. The second aim was to determine which memory variables (new learning, ABM and PSM) would explain variations in $\beta$-amyloid burden. As PSM is affected by primarily neocortical pathology, and because $\beta$-amyloid accumulation first appears in neocortical tissue, we hypothesized that PSM would be a stronger predictor of $\beta$-amyloid burden in individuals with MCI than will ABM, which is more dependent on medial temporal regions.

\section{Methods}

Participants

The Australian Imaging, Biomarkers and Lifestyle (AIBL) Study of Ageing is a longitudinal study with follow-up assessments every 18 months. This is a study of healthy controls and individuals with MCI 36 months after the larger AIBL baseline assessment. By the 36- month time- point, the cohort comprised 154 AD participants, 58 MCI participants and 611 healthy controls (HC). Funding permitted approximately one quarter of these subjects to undergo imaging at baseline. Recruitment of the AIBL cohort 
Human research approval for the current study was obtained in Victoria from St Vincent's Hospital and the University of Melbourne, and Hollywood Private Hospital in Western Australia. The methods of recruitment and exclusion criteria for the AIBL Study have been published elsewhere (Ellis et al., 2009). In brief, elderly volunteers were screened for the following exclusion criteria: a history of dementia other than DAT, psychiatric illness, Parkinson's disease, cancers within the last few years, symptomatic stroke, uncontrolled diabetes, diagnosed sleep apnoea and alcohol consumption greater than recommended levels. A diagnostic review panel of neurologists, geriatricians, psychiatrists and neuropsychologists, chaired by the fourth author (DA), oversaw the classification into HC, MCI and DAT groups according to well-established criteria (Ellis et al., 2009; Petersen et al., 1999). MCI classification was made based on cognitive performance falling at least 1.5 SD below appropriate normative levels on one or more neuropsychological tests (or two or more if initially recruited as a healthy control), expressed subjective memory complaint, and current preservation of activities of daily living. We recruited MCI participants who were still diagnosable with MCI three years after baseline AIBL diagnosis, or those with a new diagnosis of MCI by the 36-month AIBL assessment.

\section{Recruitment for this study}

This is a cross-sectional sub-study that recruited AIBL participants at the 36 month follow-up time-point of the larger AIBL study, who had specifically undergone positron emission tomography (PET) and magnetic resonance (MR) imaging at the 36-month follow-up AIBL assessment (Ellis et al., 2014). Only HC and MCI participants were eligible to participate, and $45(\mathrm{HC}=31$; $\mathrm{MCI}=14)$ consented to participate in this study. All participants were recruited via telephone and asked to participate in a one hour semi-structured interview and the CANTAB Paired Associate Learning (PAL) task in their homes. Personal memory measure 
The Episodic Autobiographical Memory Interview (EAMI) was administered in the participant's home by the first author (RB). This interview has been described in detail elsewhere (Irish et al., 2010; Irish et al., 2011b). The EAMI is a semi-structured interview that involves three parts; the first assesses PSM recall by probing personal factual information that can be shared by family and friends, and the second assesses ABM event recall by probing subjective experiences that are unique to the individual and can be recalled within a specific spatiotemporal context. The final part probed an individual's ability to reexperience the event. For the current study, a shortened version of the EAMI was used, taking approximately 40 minutes, in which recall was constrained to the Recent Period (within the last 5 years).

The PSM part involved three items. The first asked the participant to recall the names of three people that they had only met in the last five years (one point for the full name and one point for their relationship to the individual). The second involved recalling the location and route to a frequented establishment in the last five years (one point each for the name of the establishment, location, what they did there and how they travelled there). The third item related to the ability to recall the exact date, month, year and location of a personally significant event within the last five years (one point for each of the four specifics remembered). This totaled to a maximum score of 14 for the PSM component.

The ABM component required the participant to recall, in as much detail as possible, a personally significant event that occurred within the last five years. Once participants stopped spontaneously producing information pertaining to the event, the interviewer probed for further details using seven phenomenological categories taken from the Event Details Checklist of Moscovitch and colleagues (2000). These probes included event detail, temporal, spatial, sensory, implication of the event, emotion, and thought recall. Each detail was awarded a maximum score of one point, which would be summed to a maximum score of 
seven points. The autonoetic consciousness section was attached to the ABM component, and asked the individual to describe each of the following aspects within the autonoetic experience: perspective, continuity, image quality, emotional connection and recollective experience.

These interviews were recorded with the approval of the participant, transcribed, and scored by the interviewer and a blinded clinical neuropsychologist. Inter-rater reliability, as measured by intraclass correlation coefficient, was high for all three sections $\left(\mathrm{r}_{\mathrm{ABM}}=0.92\right.$; $\left.\mathrm{r}_{\mathrm{PSM}}=0.94, \mathrm{r}_{\mathrm{AUTOCON}}=0.92\right)$, aligning with reported values for the longer version of the EAMI (Irish et al., 2011b). In an effort to avoid any compensatory effects of ABM, the PSM section was administered first. Interviews were recorded with the approval of the participant, transcribed, and scored by the interviewer and a blinded clinical neuropsychologist. Both ABM and PSM recall were treated as continuous measures, while the autonoetic consciousness variables were dichotomous, i.e. an individual displayed ease or difficulty with the variable.

\section{Measures of new learning and retention}

The standard neuropsychological assessment involved a mean administration time of two hours (for details and references see Ellis et al., 2009). The following tests were administered by AIBL neuropsychologists at the 36 month time-point. The California Verbal Learning Test-Second edition (CVLT-II) short delay free recall and long delay free recall, and the Wechsler Memory Scale (WMS) Logical Memory (LM) immediate and delayed recall measures (Story 1 only) were administered to measure verbal learning, and the Rey Complex Figure Test (RCFT) 30 minute delayed recall and the CANTABeclipse v3.0 PAL stage 6 errors adjusted score, to measure nonverbal memory (participants who were unable to 
complete Stage 6 were allocated the error score of the lowest-performing individual attempting the stage).

\section{Image acquisition}

Participants in the current study had already undergone imaging in the larger AIBL study at the 36-month time-point, which we accessed. In total, 42 individuals $(\mathrm{HC}=31, \mathrm{MCI}=11)$ underwent PiB-PET or MR imaging, and 30 of these participants $(\mathrm{HC}=22, \mathrm{MCI}=8)$ underwent both PiB-PET imaging and MR. There were no demographic differences between those who underwent one or both scans so we treated these participants as the same in statistical analyses. We will now discuss the larger AIBL study imaging protocol.

\section{${ }^{11}$ C-PiB-PET imaging}

Amyloid imaging with PET was conducted using ${ }^{11} \mathrm{C}$-Pittsburgh Compound B (PiB). PET methodology has previously been described in detail (Rowe et al., 2010). Standardized uptake value (SUV) data were summed and normalized to the cerebellar cortex SUV, and the resulting tissue ratio is termed SUV ratio (SUVR). In the current study, the PiB SUVR index was considered as a continuous measure. Thirty-eight participants $(\mathrm{HC}=30, \mathrm{MCI}=8)$ underwent PiB-PET imaging.

\section{Magnetic Resonance Imaging (MRI)}

T1-weighted MRIs were segmented into cerebrospinal fluid, grey and white matter. Estimates of grey matter as well as for left and right hippocampal volumes for each participant were calculated (for detailed methods, see Rowe et al., 2010). All volumes were normalized to intracranial volume. Thirty-four participants $(\mathrm{HC}=23, \mathrm{MCI}=11)$ underwent MR imaging.

\section{Statistical analyses}


Analyses were conducted using SPSS Version 22.0. In order to reduce the potential for multicollinearity, a principal components analysis (PCA) was performed to reduce left and right hippocampal volume into one factor. The distribution of $\beta$-amyloid deposition values was skewed in HC, so a bootstrapping technique available in SPSS was used to determine a robust estimate of the mean difference between $\mathrm{HC}$ and MCI. Differences in AD imaging biomarkers were ascertained using t-tests and analysis of covariance (ANCOVA) analyses. Ttests (and chi-square analyses for the dichotomous autonoetic consciousness variables) were used to determine the difference between HC and MCI on all personal memory variables, namely, ABM, PSM, and autonoetic consciousness. Logistic regression (for autonoetic consciousness variables) and hierarchical regression (for PSM, ABM and the measures of new learning and retention) models were used to determine how $\mathrm{AD}$ imaging biomarkers relate to both personal and measures of new learning and retention, taking into account the variance explained by age and $A P O E \varepsilon 4$ carrier status. Missing data existed for both memory and affective measures but totaled less than $10 \%$ of the entire data set (refer to Table 1 ).

\section{Results}

Demographic characteristics

No significant differences between the HC and MCI groups were evident for age, sex and $A P O E \& 4$ carrier status. As expected, there was an elevated percentage of non-carriers in the HC group (71\% compared with 54\%). Healthy controls showed a trend towards having a higher percentage of individuals who attained more than 12 years of education (74\% compared with 46\%) although this did not reach statistical significance (see Table 1).

Factor analysis of left and right hippocampal volumes 
Left and right hippocampal volume were significantly correlated, $r(35)=0.86, p<0.0001$. These data were reduced in a PCA to produce one hippocampal volume factor. The loadings of both right and left hippocampal volume on the factor were 0.96 , and this factor accounted for $92.7 \%$ of the total variance explained.

\section{Hippocampal and grey matter volume}

There were no differences between HC and individuals with MCI in terms of right and left hippocampal volume or the hippocampal volume factor (see Table 1). When age was included as a covariate, it had no influence on hippocampal volume, $F(1,31)=1.56, p=$ $0.21, \eta^{2}=0.05$

There was also no significant difference in overall grey matter volume between HC and MCI. Age had a significant main effect on grey matter volume, $F(1,31)=11.43, p=0.002, \eta^{2}=$ 0.27, supporting previous findings of age-associated grey matter atrophy (Giorgio et al., 2010; Good et al., 2002). APOE \&4 carrier status did not influence any brain volume measures.

\section{Global and regional PiB SUVR}

There were no differences between HC and individuals with MCI in terms of global and regional PiB SUVR deposition (see Table 1). PiB retention was moderately variable in the two groups, and as expected, most healthy controls clustered below the PiB positive cut-off. While our healthy control group showed equivalent global PiB retention rates as reported by Rowe et al., (2010) in the 18-month follow-up, our global SUVR mean was slightly elevated in comparison with the mean PiB SUVR of 1.38 reported at 36-month follow-up (Villemagne et al., 2013). The average global PiB retention in our MCI group was below the PiB SUVR of 1.96 reported at both 18-month and 36-month time-points (Rowe et al., 2010; Villemagne et 
al., 2013). Global SUVR was highly correlated with regional SUVR values, so global PiB retention was used to represent $\mathrm{PiB}$ retention in individuals.

Age was significantly correlated with global SUVR in HC, $r(30)=0.45, p=0.02$, but not in individuals with MCI, $r(8)=0.01, p=0.98$. Having an APOE $\varepsilon 4$ allele positively correlated with global SUVR in individuals with MCI, $\rho(8)=0.76, p=0.03$, but this relationship was only a positive trend in HC, $\rho(30)=0.32, p=0.08$.

Personal memory and measures of new learning and retention

Compared to HCs, MCI participants performed worse on ABM recall but not on any other personal memory variables (perspective, continuity, image quality, emotional connection, overall recollection and PSM recall). Although individuals with MCI did not perform significantly poorer on PSM recall, a moderate effect size existed, suggesting an impact to some degree. As expected, MCI participants performed worse on all measures of new learning and retention (PAL 6 stage errors, Logical Memory immediate and delayed recall, CVLT short and long delayed recall, RCFT 30 minute delay). Table 1 shows the differences on the memory measures and their respective effect sizes.

\section{Relating AD imaging biomarkers to personal memory variables}

$\mathrm{AD}$ imaging biomarkers, age and $A P O E$ \&4 were not associated with autonoetic consciousness variables (i.e. perspective, continuity, image quality, emotional connection and overall recollection, refer to Table 2 for details). The data for emotional connection were highly skewed so this variable was excluded from analyses. At a univariate level, ABM recall showed a trend towards being related to global $\operatorname{SUVR}(\beta=-0.39, p=0.06)$ and had a significant relationship with APOE $\varepsilon 4(\beta=0.43, p=0.04)$, but the overall model was not significant $(p=0.25)$. A trend between poorer ABM recall and smaller hippocampal volume 
in MCI participants showed a moderate effect size, this was not significant. PSM recall was significantly related to global $\mathrm{PiB}$ retention $(\beta=-0.62, p=0.001)$ after taking into account the variance explained by age, $A P O E \& 4$ carrier status, grey matter and hippocampal volume (see Table 3). Figure 1 shows the strong relationship between poorer PSM recall and larger global $\mathrm{PiB}$ retention in both the $\mathrm{HC}$ and $\mathrm{MCI}$ groups, although the relationship was not significant in individuals with MCI.

Memory predictors of $A D$ biomarkers: a final model

In order to reduce the number of variables in a post-hoc regression analysis, PCA with varimax rotation was run on the measures of new learning and retention (PAL stage 6 errors, Logical Memory immediate and delayed recall, and CVLT short and long delayed recall). RCFT 30 minute delayed recall was excluded from the analysis as it produced a Hayward case (or a loading greater than 1). Two memory factors with Bartlett factor scores were produced, accounting for $83 \%$ of the variance explained; the first factor incorporated the CVLT-II and PAL measures, and the second factor included the Logical Memory measures.

A post-hoc step-wise regression model was conducted to find the best statistical predictor (out of ABM, PSM, the two memory factors, age and APOE ع4) of global PiB retention in HC and those with MCI (see Table 3, Model 9). The best predictor was PSM recall $(\beta=-0.59, p=0.001)$, followed by APOE $\varepsilon 4(\beta=0.41, p=0.02)$. This overall model accounted for $40 \%$ of the variance explained in global $\mathrm{PiB}$ retention.

\section{Discussion}

Autobiographical memory recall was impaired in individuals with MCI, supporting previous research (Hou et al., 2005; Irish et al., 2011a; Irish et al., 2010; Leyhe et al., 2009). Performance in PSM recall did not differentiate the diagnostic groups, but the moderate effect size is commensurate with that shown in our previous study (Buckley et al., 2014). 
Participants with MCI did not differ from controls on the overall autonoetic re-experiencing of a memory, consistent with a previous investigation using the same measure (Irish et al., 2010). Unlike Irish and colleagues (Irish et al., 2010), we did not find differences between the groups in emotional connection, continuity of the imagery, viewer perspective and vividness, most likely reflecting different in patient samples and study methodology. Aligning with the established profile for amnestic MCI (Winblad et al., 2004), participants with MCI were impaired on all measures of new learning and retention, namely Logical Memory, CVLT-II, RCFT and the PAL task. Inclusion in the MCI group was dependent on performance at least 1.5SD below the normative mean on any one of the first three measures (or other AIBL measures not reported here). While this introduces some potential for circularity, the PAL task, which played no role in defining the groups, also differentiated HC from MCI.

There were no differences in global neocortical amyloid burden or grey matter volumes between healthy controls and those with MCI. The healthy control group showed global PiB retention comparable to that observed in previous AIBL studies, but the average $\mathrm{PiB}$ retention for our MCI participants was lower than the larger AIBL cohort, at 1.7 compared with 1.9 (Rowe et al., 2010; Villemagne et al., 2013). The present cohort is derived from the Villemagne et al. (2013) study, in the context of the longitudinal AIBL study. Individuals with a longer history of cognitive decline, who had since converted to diagnosable dementia, who in their majority presented with high $\mathrm{PiB}$ retention, did not meet selection criteria for the present study, yielding a cohort with low PiB retention. We also found a large dispersion of grey matter volumes in both diagnostic groups, similar to that seen in the larger AIBL cohort (Rowe et al., 2010; Villemagne et al., 2013).

Personal memory, specifically, PSM, ABM and autonoetic consciousness, was not related to overall grey matter or hippocampal volumes. There was a trend towards a 
relationship between smaller hippocampal volume and lower ABM performance in individuals with MCI, but this did not reach statistical significance. In functional imaging studies of healthy adults, ABM is associated with a 'core' functional network, featuring the hippocampus (for a review, see Maguire, 2001; Svoboda et al., 2006). Hippocampal atrophy did not have a relationship with ABM in either diagnostic category, raising the notion that different samples and imaging methodologies might play a role in the divergent finding. It is also possible that through the course of a 36-month longitudinal study, our sample contained a higher percentage of individuals with non-progressive MCI, which might explain the lack of an expected finding. An alternative explanation is that this might be a slightly more resilient group, and the data might be considered in this light.

Global neocortical amyloid burden had a significant impact on PSM recall in healthy controls. A correlation of moderate size was also evident in the MCI group, but fell short of significance due to small sample size. Our findings align with studies showing verbal memory dysfunction in amyloid positive non-demented elderly (Lim et al., 2013b; Pike et al., 2007; Rentz et al., 2011). This relationship between PSM and neocortical amyloid deposition was not changed by controlling for age, grey matter volume, and $A P O E \varepsilon 4$ carrier status, suggesting a slight divergence from some studies showing a moderating effect of APOE $\varepsilon 4$ (Kantarci et al., 2012; Lim et al., 2013a). PSM performance was the sole personal memory variable associated with brain neocortical amyloid burden in the final model, contributing unique variance to $\mathrm{PiB}$ retention, even when measures of new learning and retention were included. Interestingly, PSM did not differentiate HC from MCI. Unlike PSM, ABM recall did differentiate between the diagnostic categories but did not demonstrate a convincing relationship with neocortical amyloid burden. This pattern raises the possibility that $A B M$ difficulties are underpinned by an alternative neuropathological mechanism, such as tauopathy. The autonoetic re-experiencing of personal memories, which is fundamental to 
ABM function (Conway \& Pleydell-Pearce 2000; Wheeler et al., 1997), was also not associated with neocortical amyloid deposition.

\section{Conclusion}

While the samples in this study are smaller than desirable, these findings do open up what we consider to be an important new direction in Alzheimer's research. To the best of our knowledge, this study provides the first insight into the impact of early AD-related neuropathological changes on personal memory function in the non-demented elderly. Our findings show that ABM and PSM were differentially affected by diagnostic category. Neocortical amyloid deposition affected both systems disparately by influencing PSM but not ABM. These findings suggest that separate neuropathological mechanisms are mediating $\mathrm{ABM}$ and PSM. ABM and PSM are largely dependent on different, but overlapping and interacting, neurofunctional components of the temporal system (Gilboa et al., 2004; Maguire 2001; Svoboda et al., 2006). At an explanatory level, the notion that while PSM is influenced by neocortical amyloid burden, other underlying neurodegenerative processes that initially impinge on the hippocampal formation, such as tauopathy, might exert a greater effect on the essentially episodic aspects of autobiographical memory.

\section{Conflict of Interest}

None.

\section{Description of authors' roles}

Rachel Buckley administered the EAMI interview, carried out the statistical analyses and wrote the paper. Michael Saling oversaw the analyses. David Ames, Alan Rembach, Stephanie Rainey-Smith and Kathryn Ellis oversee and coordinate the AIBL study at the Melbourne site. David Ames also chairs the AIBL diagnostic panel, on which Nicola Lautenschlager, Greg Savage, Cassandra Szoeke and Kathryn Ellis also serve. Nicola Lautenschlager, Paul Maruff and Greg Savage serve on the leadership group of the Clinical 
and Cognitive Stream of the AIBL study. Lance Macauley represented CSIRO on the management committee. Ralph Martins and Colin Masters oversee the AIBL study, sit on the management committee. Ralph Martins and Stephanie Rainey-Smith lead and coordinate the Perth arm of the AIBL study. Chris Rowe leads the AIBL imaging arm, serves on the AIBL management committee. Victor Villemagne also oversaw the imaging component. Cassandra Szoeke represented CSIRO at management committee meetings from 2009-2011. All authors critically reviewed drafts of this manuscript.

\section{Acknowledgements including sources of support}

The AIBL study (www.AIBL.csiro.au) received support from CSIRO, the Science and Industry Endowment Fund (www.SIEF.org.au) NHMRC and Dementia Collaborative Research Centres (DCRC), as well as Industry, including Pfizer, Merck, Janssen and GE Healthcare. We thank all the participants who took part in the study and the clinicians who referred MCI participants to the study. 


\section{References}

Buckley, R., et al. (2013) Factors affecting subjective memory complaints in the AIBL aging study: biomarkers, memory, affect, and age. International Psychogeriatrics, 25(8), 1307-1315. doi: $10.1017 / \mathrm{S} 1041610213000665$

Buckley, R., et al. (2014) Personal memory function in mild cognitive impairment (MCI) and subjective memory complaints: Results from the Australian Imaging, Biomarkers, and Lifestyle (AIBL) Study of Ageing Journal of Alzheimer's Disease, 40(3).

Conway, M. A. and Pleydell-Pearce, C. W. (2000) The construction of autobiographical memories in the self-memory system. Psychological Review, 107(2), 261. doi: 10.1037//0033295X.107.2.261

Ellis, K., et al. (2009) The Australian Imaging, Biomarkers and Lifestyle (AIBL) study of aging: methodology and baseline characteristics of 1112 individuals recruited for a longitudinal study of Alzheimer's disease. International Psychogeriatrics, 21(4), 672-87. doi: 10.1017/S1041610209009405

Ellis, K., et al. (2014) Rates of diagnostic transition and cognitive change at 18-month follow-up among 1,112 participants in the Australian Imaging, Biomarkers and Lifestyle Flagship Study of Ageing (AIBL). International Psychogeriatrics, 26(4), 543-554. doi: 10.1017/S1041610213001956

Fraser, L. M., O'Carroll, R. E., and Ebmeier, K. P. 2008. The effect of electroconvulsive therapy on autobiographical memory: a systematic review. The journal of ECT, 24, 10-17. doi: 10.1097/YCT.0b013e3181616c26

Gilboa, A., Ramirez, J., Köhler, S., Westmacott, R., Black, S. E. and Moscovitch, M. (2005) Retrieval of autobiographical memory in Alzheimer's disease: Relation to volumes of medial temporal lobe and other structures. Hippocampus, 15(4), 535-550. doi: 10.1002/hipo.20090

Gilboa, A., Winocur, G., Grady, C. L., Hevenor, S. J. and Moscovitch, M. (2004) Remembering our past: functional neuroanatomy of recollection of recent and very remote personal events. Cerebral Cortex, 14(11), 1214-1225. doi: 10.1093/cercor/bhh082

Giorgio, A., et al. (2010) Age-related changes in grey and white matter structure throughout adulthood. Neuroimage, 51(3), 943-951. doi: 10.1016/j.neuroimage.2010.03.004

Good, C. D., Johnsrude, I. S., Ashburner, J., Henson, R. N., Fristen, K. and Frackowiak, R. S. (2002) A voxel-based morphometric study of ageing in 465 normal adult human brains. in Biomedical Imaging, 2002. 5th IEEE EMBS International Summer School on: IEEE. 16

Hou, C. E., Miller, B. L. and Kramer, J. H. (2005) Patterns of autobiographical memory loss in dementia. International Journal of Geriatric Psychiatry, 20(9), 809-815. doi: 10.1002/gps.1361

Irish, M., Lawlor, B., Coen, R. and O'Mara, S. (2011a) Everyday episodic memory in amnestic Mild Cognitive Impairment: a preliminary investigation. BMC Neuroscience, 12(1), 80. doi: $10.1186 / 1471-2202-12-80$

Irish, M., Lawlor, B. A., O'Mara, S. M. and Coen, R. F. (2010) Exploring the recollective experience during autobiographical memory retrieval in amnestic mild cognitive impairment. Journal of the International Neuropsychological Society, 16(3), 546-55. doi: 10.1017/S1355617710000172 
Irish, M., Lawlor, B. A., O'Mara, S. M. and Coen, R. F. (2011b) Impaired capacity for autonoetic reliving during autobiographical event recall in mild Alzheimer's disease. Cortex, 47, 236249.doi: 10.1016/j.cortex.2010.01.002

Jessen, F., et al. (2014) AD dementia risk in late MCI, in early MCI, and in subjective memory impairment. Alzheimer's \& Dementia, 10(1), 76-83.doi: 10.1016/j.jalz.2012.09.017

Kantarci, K., et al. (2012) APOE modifies the association between A $\beta$ load and cognition in cognitively normal older adults. Neurology, 78(4), 232-240. doi: 10.1212/WNL.0b013e31824365ab

Leyhe, T., Müller, S., Milian, M., Eschweiler, G. W. and Saur, R. (2009) Impairment of episodic and semantic autobiographical memory in patients with mild cognitive impairment and early Alzheimer's disease. Neuropsychologia, 47(12), 2464.doi: 10.1016/j.neuropsychologia.2009.04.018

Lim, Y. Y., et al. (2013a) A $\beta$ amyloid, cognition, and APOE genotype in healthy older adults. Alzheimer's \& Dementia, 9(5), 538-545. doi: 10.1016/j.jalz.2012.07.004

Lim, Y. Y., et al. (2013b) Effect of amyloid on memory and non-memory decline from preclinical to clinical Alzheimer's disease. Brain. doi: 10.1093/brain/awt286

Maguire, E. A. (2001) Neuroimaging studies of autobiographical event memory. Philosophical Transactions of the Royal Society of London. Series B: Biological Sciences, 356(1413), 1441-1451. doi: 10.1098/rstb.2001.0944

Moscovitch, M., Yaschyshyn, T., Ziegler, M., Nadel, L., and Tulving, E. (Ed). (2000) Remote episodic memory and retrograde amnesia: Was Endel Tulving right all along? Memory, consciousness, and the brain: The Tallinn Conference, (pp. 331-345). New York: Psychology Press.

Mormino, E. C., et al. (2009) Episodic memory loss is related to hippocampal-mediated $\beta$-amyloid deposition in elderly subjects. Brain, 132(5), 1310-1323. doi: 10.1093/brain/awn320

Petersen, R. C., Smith, G. E., Waring, S. C., Ivnik, R. J., Tangalos, E. G. and Kokmen, E. (1999) Mild cognitive impairment: clinical characterization and outcome. Archives of Neurology, 56(3), 303-8. doi: 10.1001/archneur.56.3.303

Pike, K. E., et al. (2011) Cognition and beta-amyloid in preclinical Alzheimer's disease: Data from the AIBL study. Neuropsychologia, 49(9), 2384-2390. doi: 10.1016/j.neuropsychologia.2011.04.012

Pike, K. E., et al. (2007) \{beta $\}$-amyloid imaging and memory in non-demented individuals: evidence for preclinical Alzheimer's disease. Brain, 130(11), 2837-2844. doi: 10.1093/brain/awm238

Rentz, D. M., et al. (2011) Face-name associative memory performance is related to amyloid burden in normal elderly. Neuropsychologia, 49(9), 2776-2783. doi: 10.1016/j.neuropsychologia.2011.06.006

Robinson J. A. and Swanson K. L. (1990) Autobiographical memory: The next phase. Applied Cognitive Psychology, 4, 321-335. doi: 10.1002/acp.2350040407

Rowe, C. C., et al. (2010) Amyloid imaging results from the Australian Imaging, Biomarkers and Lifestyle (AIBL) study of aging. Neurobiology of Aging, 31(8), 1275-83.doi: 10.1016/j.neurobiolaging.2010.04.007 
Sperling, R. A., et al. (2010) Criteria for preclinical Alzheimer's disease. Accessed from: http://www.alz.org/research/diagnostic_criteria/preclinical_recommendations.pdf

Svoboda, E., McKinnon, M. C. and Levine, B. (2006) The functional neuroanatomy of autobiographical memory: A meta-analysis. Neuropsychologia, 44(12), 2189-2208.doi: 10.1016/j.neuropsychologia.2006.05.023

Villemagne, V. L., et al. (2013) Amyloid $\beta$ deposition, neurodegeneration, and cognitive decline in sporadic Alzheimer's disease: a prospective cohort study. The Lancet Neurology, 12(4), 357367. doi: 10.1016/S1474-4422(13)70044-9

Wheeler, M. A., Stuss, D. T. and Tulving, E. (1997) Toward a theory of episodic memory: the frontal lobes and autonoetic consciousness. Psychological Bulletin, 121(3), 331-54.doi: 10.1037//0033-2909.121.3.331

Winblad, B., et al. (2004) Mild cognitive impairment - beyond controversies, towards a consensus: report of the International Working Group on Mild Cognitive Impairment. Journal of Internal Medicine, 256(3), 240-246. doi: 10.1111/j.1365-2796.2004.01380.x 